\title{
Role of 3-D Conventional Angiography In Evaluation of Intra Carnial Aneurysms
}

\author{
Ahmed M Bassiouny*, Maher M Arafa*, Sameh M Abdelwahab*, \\ Motaaz M Albeblawy *, Amr M Abdelsamad*. \\ *Department of Radio-diagnosis, Faculty of Medicine, Ain shams University
}

\begin{abstract}
Introduction: intracranial aneurysms are pathological enlargement of the brain arteries that are most commonly located in the circle of Willis. Intracranial aneurysms are relatively common with a prevalence of approximately $4 \%$. The real danger of aneurysms is subarachnoid hemorrhage. 3D digital subtraction angiography has become a critical imaging tool in neuroradiology allowing for the visualization of detailed cerebral vasculature prior to any intervention.
\end{abstract}

Aim of the work: The aim of this work is to evaluate the diagnostic performance of 3D conventional angiography in the evaluation of intracranial aneurysms compared with the conventional cerebral angiography.

Methods: The studied group included 20 patients (5 men and 15 women) with subarachnoid hemorrhage or known to have cerebral aneurysms. All patients were subjected to conventional cerebral angiography and 3D cerebral angiography, using C-arm (Toshiba) rotational technique.

Results: 3D digital subtraction angiography is superior to conventional digital subtraction angiography in $100 \%$ of the cases 3D imaging revealed the proper aneurysmal shape, size, precise assessment of its neck and relation to the surrounding vessels.

Conclusion: Three-dimensional DSA improves the detection and delineation of intracranial aneurysms

KEY WORDS: 3-D Conventional Angiography , Intra Carnial Aneurysms.

\section{INTRODUCTION}

Intracranial aneurysms are pathological enlargement of brain arteries that are most commonly located in the circle of Willis ${ }^{(\mathbf{1})}$.

The incidence of subarachnoid hemorrhage (SAH) due to rupture aneurysm is 10$25: 100,000$ and is a devastating event associated with high morbidity and mortality from rebleeding and vasospasm ${ }^{(2)}$.

3D digital subtraction angiography (3D DSA) has demonstrated its clinical efficacy in precise depiction of the aneurysmal neck and its relationship to adjacent vessels. Moreover, it has been shown that 3D DSA reveals aneurysms not seen with conventional digital subtraction angiography (DSA) ${ }^{(3)}$. These features are important to consider when one decides surgical or endovascular treatment ${ }^{(4)}$.

\section{THE AIM OF THE WORK}

Is to assess the diagnostic performance of 3D conventional angiography in the evaluation of intracranial aneurysms compared with the conventional cerebral angiography

METHODS

3D conventional cerebral angiography was performed over a period of 2 years on 20 patients with subarachnoid hemorrhage or known to have cerebral aneurysms 5men, 15 women; age range 30-70years with cerebral aneurysms and variable clinical presentations, the most common one was headache (18 cases) 15 after subarachnoid hemorrhage. Other complains include eye pain and visual deficits (2 cases).Some of our Patients had pre-procedural investigations including PT, PTT, INR and renal function tests.

After taking consents from all patients or their first degree relatives in unconscious and emergency cases, patients usually fast 48 hours before the procedure but are permitted to take any regular medications (particularly antihypertensive \& antiplatelet medications) orally with a little water. Patients receiving long term anticoagulation should discontinue warfarin 6 hours prior to the procedure to decrease the risk of punctural hematoma. Similarly, patients with preexistent renal disease are admitted on the prior day for intravenous hydration. 
Access to the cerebral circulation was done through a femoral puncture after groin sterilization; conventional angiography at site of aneurysm was performed first, followed by 3D angiography.

Three dimensional data sets are obtained from rotational series consisting of two rotations. The first provides the subtraction mask. The $\mathrm{C}-$ arm is rotated 200 degrees within 5 seconds with an exposure rate of 8.8 frames per second .A total of 44 images with a 512 x 512 matrix are acquired .The second rotation then performed during the administration of contrast material. All 88 images are transferred to a workstation via a network, subtraction images and a primary 3D model are generated within 8 minutes (1 minute for transfer, 7 minutes for reconstruction).then images are analyzed by a neuroradiologist.

\section{RESULTS}

Our series included 20 patients, 5men, 15 women; age range 30-70 years (fig. 1) with cerebral aneurysms and other complains as eye pain and visual deficits ( 2 cases), our cases can be grouped as in figure (fig. 2).

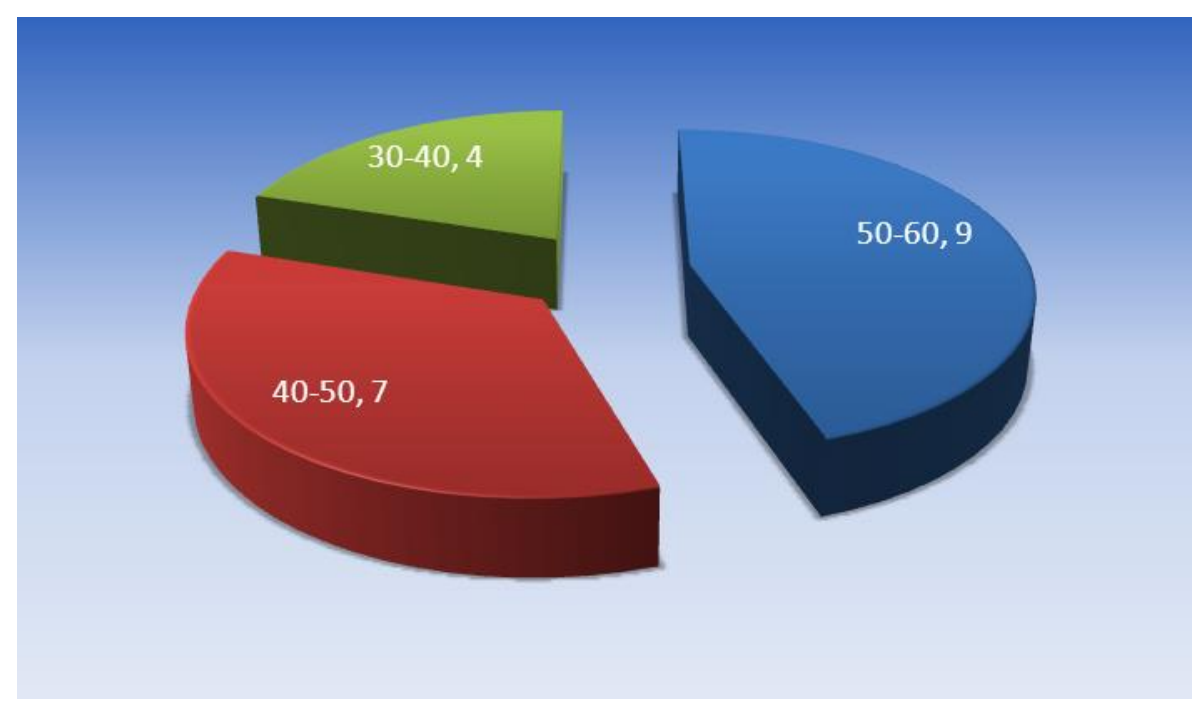

Fig. (1): Distribution of cases according to age group

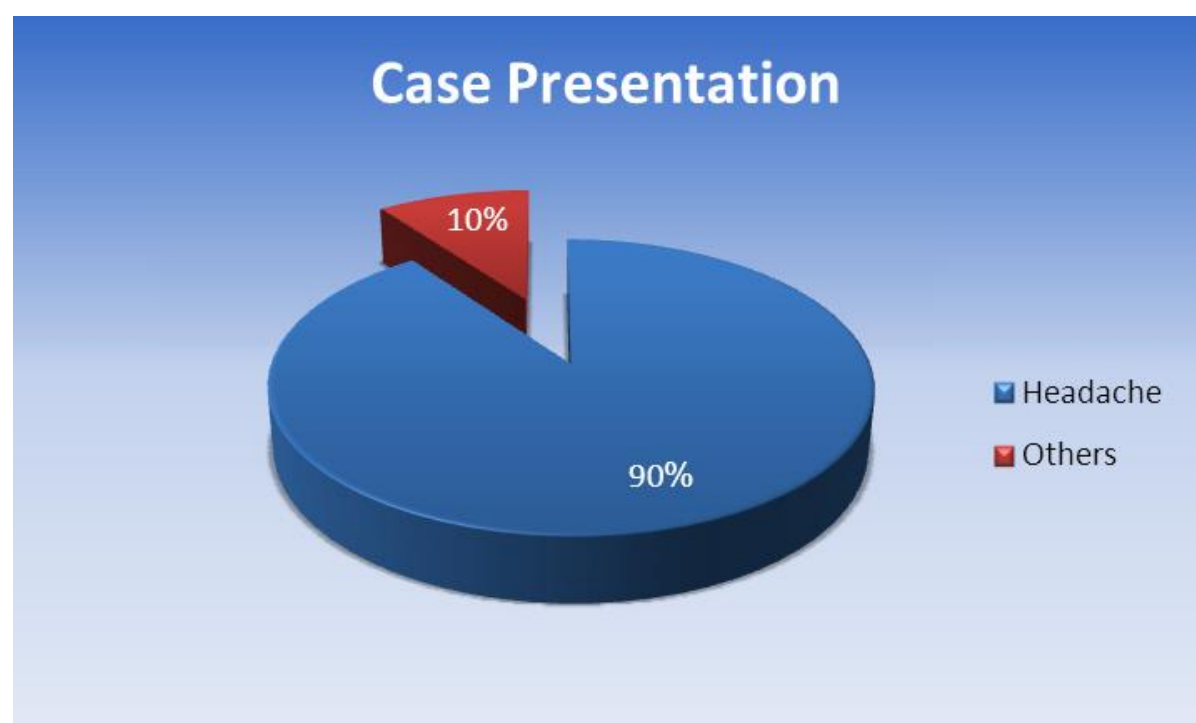

Fig. (2): Clinical presentation.

In our study 8 aneurysms were found in the middle cerebral artery, 6 in the internal carotid, 4 in the anterior communicating artery and 2 in the basilar artery. 


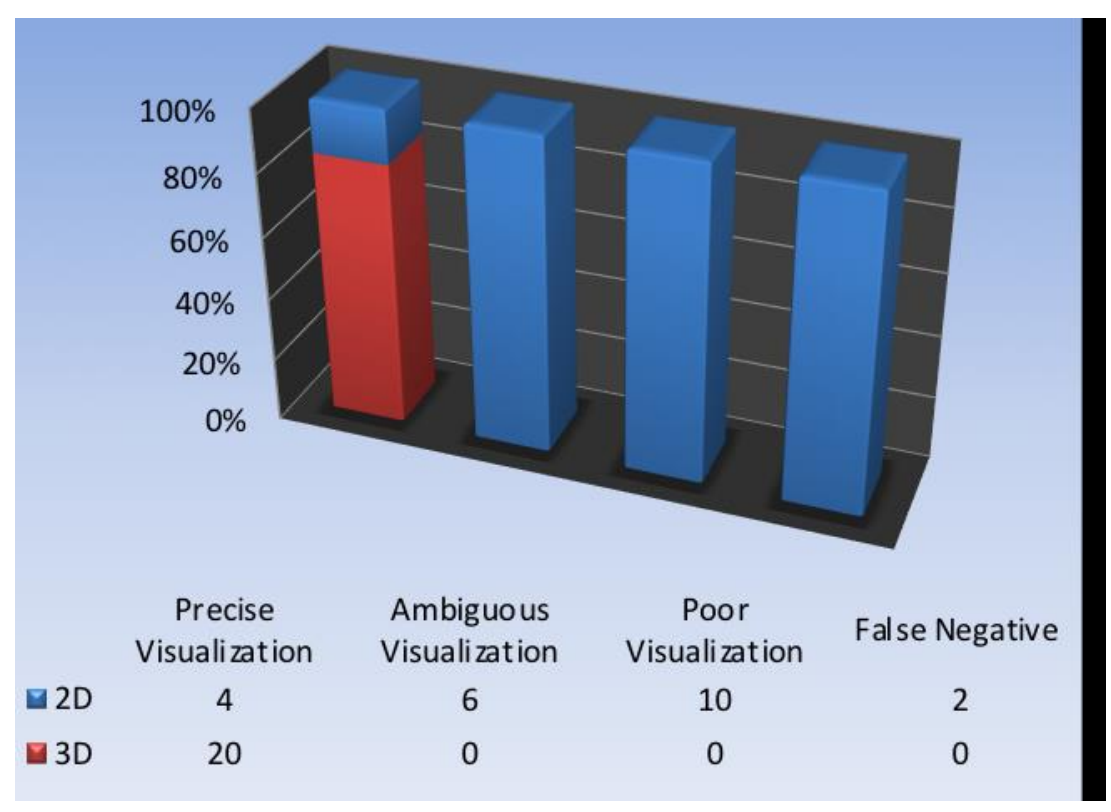

Fig. (3): Shows comparison between 2D and 3D angiography results.

Regarding proper visualization of the aneurysms, In 10 cases out of the $20(50 \%)$, vessel incorporation, into the neck and relation to the surrounding vessels were poorly visualized by using the $2 \mathrm{D}$ angiography technique.

In only 4 patients of the $20(20 \%), 2 \mathrm{D}$ angiography allowed precise visualization of the aneurysms without ambiguity, while 6 cases (30\%) gave ambiguous results by $2 \mathrm{D}$ angiography.

In 3D DSA images, it was always possible to find one or more orientations that delineated the aneurysm neck from the parent artery and near by vessels.

Of the 3D images, none led to poor visualization or misinterpretation of the neck. Two false negative results were noted at $2 \mathrm{D}$ angiography, while $3 \mathrm{D}$ angiography of these patients was able to detect the aneurysms precisely fig. (3).

Table (1): Visualization of location and aneurysmal neck at 2D and 3D DSA.

\begin{tabular}{|l|r|r|r|r|}
\hline $\begin{array}{c}\text { neck visualization } \\
\text { and location }\end{array}$ & 2D & 3D & \multicolumn{2}{c|}{ Chi-square } \\
& DSA & DSA & $X^{2}$ & P-value \\
\cline { 3 - 5 } & & 20 & 26.60 & $<0.001^{*}$ \\
\hline Precise visualization & 6 & 0 & 7.05 & $0.008^{*}$ \\
\hline Ambiguous visualization & 10 & 0 & 13.3 & $<0.001^{*}$ \\
\hline Poor visualization $\backslash$ misinterpret & 6 & & & \\
\hline
\end{tabular}

Non sig. $>0.05$ Sig. $<0.05$ High sig. $<0.001 *$

In 2D angiography the aneurysm shape was precisely assessed in four (20\%) of 20 aneurysms and poorly assessed in five (25\%) at biplanar 2D DSA; visualization was ambiguous in $11(55 \%)$ aneurysms. In contrast, 3D imaging clearly demonstrated the shape and was effective in depicting the complex bilobated or irregular shapes of all the aneurysms. Thus, 3D was the only imaging mode of those studied that enabled precise visualization of the aneurysm shape in all cases. 
Table (2): Aneurysm shape.

\begin{tabular}{|c|c|c|c|c|c|}
\hline \multirow{3}{*}{\multicolumn{2}{|c|}{ Aneurysm Shape }} & \multicolumn{4}{|c|}{ DSA } \\
\hline & & \multicolumn{2}{|c|}{$2 \mathrm{D}$} & \multicolumn{2}{|c|}{$3 \mathrm{D}$} \\
\hline & & $\mathrm{N}$ & $\%$ & $\mathrm{~N}$ & $\%$ \\
\hline \multicolumn{2}{|c|}{ Precise visualization } & 4 & 20.0 & 20 & 100.0 \\
\hline \multicolumn{2}{|c|}{ Ambiguous visualization } & 11 & 55.0 & 0 & 0.0 \\
\hline \multicolumn{2}{|c|}{ Poor visualization } & 5 & 25.0 & 0 & 0.0 \\
\hline \multicolumn{2}{|l|}{ Total } & 20 & 100.0 & 20 & 100.0 \\
\hline \multirow{2}{*}{ P-value } & $\mathrm{X} 2$ & \multicolumn{4}{|c|}{26.667} \\
\hline & P-value & \multicolumn{4}{|c|}{$<0.001 *$} \\
\hline
\end{tabular}

\section{DISCUSSION}

Three-dimensional reconstruction of intracranial vessels at imaging is of particular interest in the evaluation of aneurysms, especially in complex cases. Precise visualization of the aneurysm neck and its relationships with parent vessels are fundamental factors that must be assessed before choosing between an endovascular and neurosurgical treatment. The shape and size of the aneurysm also are essential to know before performing endovascular occlusion with coils. This information can be obtained in part using computed tomographic angiography (CT) and magnetic resonance (MR) angiography ${ }^{(5)}$.

Compared with arterial digital subtraction angiography (DSA), however, these techniques have inferior spatial resolution, have lower sensitivity in the detection of small $\quad(<3 \mathrm{~mm}) \quad$ intracranial aneurysms ${ }^{(5)}$.

Results of the present work showed excellent images and adds the third dimension to the conventional angiography. We agree with the authors Hochmuth et al. and Sugahara et al. ${ }^{(5)}{ }^{(6)}$ that 3D rotational angiography is highly accurate and can improve both detection rate and the anatomic depiction of intracranial aneurysms

For endovascular treatment, precise analysis of the aneurysm anatomy and a working view that delineates the neck from adjacent vessels are required. For this purpose 3D angiography have been shown to provide additional information to conventional angiographic findings for surgical planning for intracranial aneurysms ${ }^{(6)}$.

In the current work the aneurysm neck and relationship to surrounding vessels were significantly better demonstrated on 3D DSA than on 2D DSA. Similar results were obtained from Congard et al. and Brinjikii et al. ${ }^{(8)(9)}$.

In the current work, we demonstrated differences between aneurysmal size measurements obtained using 3D-DSA and 2DDSA. Results of the present study showed that 3D DSA is more sensitive in detecting small aneurysms than the conventional 2D DSA, despite its lower spatial resolution. These results were consistent with those obtained by Van Rooij et al., and Ishihara et al. (10) (11).

\section{Case (1)}

60 year old female patient, having subarachnoid hemorrhage, performed 2D conventional digital subtraction angiography revealing a basilar trunk bisaccular aneurysm near the origin of the superior cerebellar artery followed by 3D subtraction angiography, the neck could not be identified by 2D study, while it was clearly seen by 3D study, the aneurysmal sac measured $6 \times 5 \mathrm{~mm}$ and the neck measured $2.5 \mathrm{~mm}$. 
Fig. (5): Patient with bisaccular basilar trunk aneurysm near the origin of left superior cerebellar artery
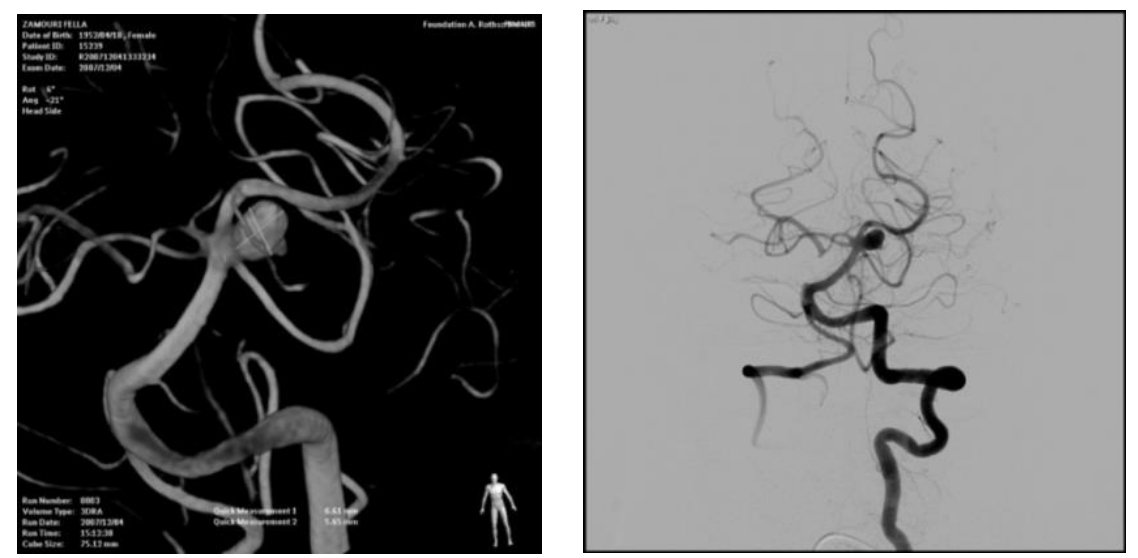

\section{Case (2)}

35 year old male with basilar tip aneurysm . 2D angiography was unable to precisely detect the neck. The aneurysmal neck was detected on 3D angiography measuring $20 \mathrm{~mm}$, and 40x $38 \mathrm{~mm}$ maximum diameter of the aneurysm sac.

$3 \mathrm{D}$ angiography showed the relation between the aneurysm and both posterior cerebral arteries, and that its neck is not extending to either of them.

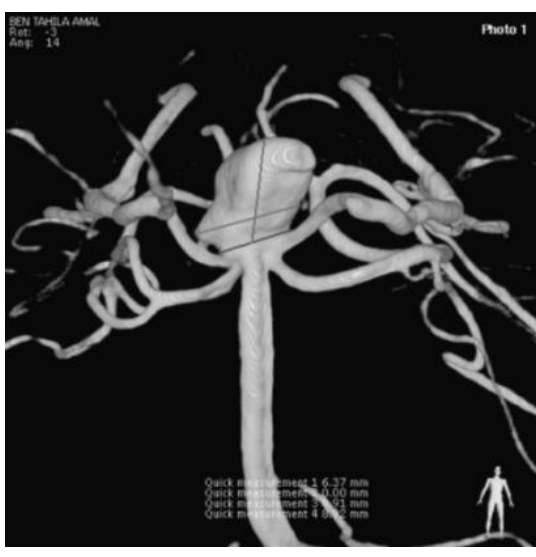

Fig. (6): 3D cerebral angiography showing basilar tip aneurysm neck measuring $20 \mathrm{~mm}$ and aneurysmal sac measuring 40 x38 mm

\section{CONCLUSION}

Three-dimensional DSA improves the detection and delineation of intracranial aneurysms,

The size of an aneurysm and the diameter of the neck can be correctly measured using 3D-DSA. The size of the aneurysm neck on 3D DSA tended to be larger than on 2D DSA.

We agree that $3 \mathrm{D}$ DSA is superior to $2 \mathrm{D}$ DSA in determination of aneurysm detection rates as well as depiction of aneurysm shape, size, neck location, and relationship to the parent artery.

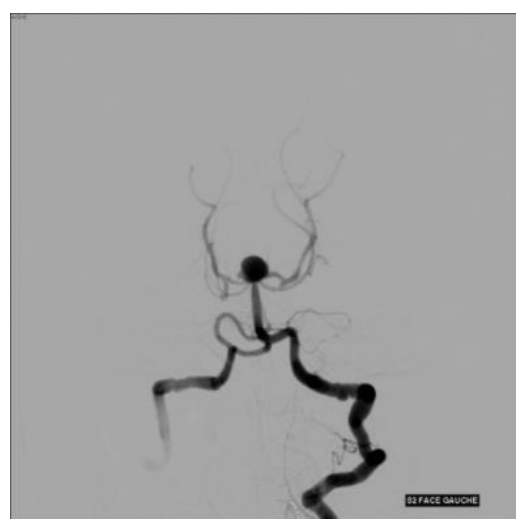

Fig. (7): 2D cerebral angiography A.P. and lateral views showing a basilar tip aneurysm with no definite of neck measurement.

\section{REFERENCES}

(1)Weir B, (2002): Unruptured intracranial aneurysms: A review, J. Neurosurg, vol. 96, pp. 3-42

(2) Maira G, Albnese A, Pentimalli L et al., (2006): Treatment of intracranial aneurysms. Clin Exp hypertens 28:371-376

(3) Hochmuth A., Spetzger U., Schumacher M. (2002): Comparison of three dimensional rotational angiography with digital subtraction angiography in ruptured cerebral aneurysms. AJNR Am J Neuroradiol, 23:1199-205

(4) Anxionnat R, Blacard S, Ducrocq X, et al. (2001): Intracranial aneurysms: clinical value of 3D 
digital subtraction angiography in the therapeutic decision and endovascular treatment .Radiology, 3 :799-808

(5) Korogi Y, Takahashi M, Katada K, et al. (1999): Intracranial aneurysms: detection with three-dimensional CT angiography with volume rendering-comparison with conventional angiographic and surgical findings. Radiology; 211:497-506.

(6) Sugahara T, Korogi Y, Nakashima K, et al. (2002): Comparison of 2D and 3D digital subtraction angiography in evaluation of intracranial aneurysms. Am J Neuroradiol.; 23:1545-52.(7) Wolfe SQ, Baskaya MK, Heros RC, Tummala RP (2006): Cerebral Aneurysms: Learning from the Past and Looking toward the Future (chapter 16): Clin Neurosurg 53:157-178.
(8) Cognard C, Weill A, Castaings L, Rey A, Moret J. (1988): Intracranial berry aneurysms: angiographic results after endovascular treatment. Radiology; 206: 499-510.

(9) Brinjikji W, Cloft H, Lanzino G, et al. (2009): Comparison of 2D digital subtraction angiography and $3 \mathrm{D}$ rotational angiography in the evaluation of dome-to-neck ratio. Am J Neuroradiol; 30:831-34. (10) van Rooij WJ, Sprengers ME, de Gast AN, et al. (2008): 3D rotational angiography: the new old standard in the detection of additional intracranial aneurysms. Am J Neuroradiol.; 29:976-79.

(11) Ishihara H, Kato S, Akimura T, et al. (2007): Angiogram-negative subarachnoid hemorrhage in the era of three dimensional rotational angiography. J Clin Neurosci; 14:252-55. 